\title{
DNA hypermethylation enhanced telomerase reverse transcriptase expression in human-induced pluripotent stem cells
}

\author{
Ken Takasawa ${ }^{1} \cdot$ Yoshikazu Arai $^{1} \cdot$ Mayu Yamazaki-Inoue $^{2} \cdot$ Masashi Toyoda $^{3} \cdot$ \\ Hidenori Akutsu ${ }^{2} \cdot$ Akihiro Umezawa $^{2} \cdot$ Koichiro Nishino ${ }^{1,4}$
}

Received: 3 October 2017 / Accepted: 26 October 2017 / Published online: 4 November 2017

(c) The Author(s) 2017. This article is an open access publication

\begin{abstract}
During reprogramming into human induced pluripotent stem cells (iPSCs), several stem cell marker genes are induced, such as $O C T-4, N A N O G, S A L L 4$, and TERT. OCT-4, NANOG, and SALL4 gene expression can be regulated by DNA methylation. Their promoters become hypomethylated in iPSCs during reprogramming, leading to their induced expression. However, epigenetic regulation of the TERT gene remains unclear. In this study, we focused on epigenetic regulation of the human TERT gene and identified a differentially methylated region (DMR) at a distal region in the TERT promoter between human iPSCs and their parental somatic cells. Interestingly, the TERT-DMR was highly methylated in iPSCs, but low-level methylation was observed in their parental somatic cells. Region-specific, methylatedpromoter assays showed that the methylated TERT-DMR up-regulated the promoter activity in iPSCs. In addition, Lamin B1 accumulated at the TERT-DMR in iPSCs, but
\end{abstract}

Electronic supplementary material The online version of this article (doi:10.1007/s13577-017-0190-x) contains supplementary material, which is available to authorized users.

Koichiro Nishino

aknishino@cc.miyazaki-u.ac.jp

1 Laboratory of Veterinary Biochemistry and Molecular Biology, Graduate School of Medicine and Veterinary Medicine/Faculty of Agriculture, University of Miyazaki, 1-1 Gakuen-Kibanadai-Nishi, Miyazaki 889-2192, Japan

2 Center for Regenerative Medicine, National Research Institute for Child Health and Development, 2-10-1 Okura, Setagaya-ku, Tokyo 157-8535, Japan

3 Research Team for Geriatric Medicine (Vascular Medicine), Tokyo Metropolitan Institute of Gerontology, 35-2 Sakaemachi, Itabashi-ku, Tokyo 173-0015, Japan

4 Center for Animal Disease Control, University of Miyazaki, 1-1 Gakuen-Kibanadai-Nishi, Miyazaki 889-2192, Japan not in their parent somatic cells. These results suggested that the TERT transcription was enhanced by DNA methylation at the TERT-DMR via binding to nuclear lamina during reprogramming. Our findings shed light on a new functional aspect of DNA methylation in gene expression.

Keywords Human TERT gene · Human iPSCs · DNA methylation · Epigenetics $\cdot$ Lamin B1 $\cdot$ Reprogramming

\section{Introduction}

Telomerase reverse transcriptase (TERT) is a key component of telomerase and an important molecule for stem cells. TERT elongates telomere sequences at the ends of chromosomes and defines the high proliferative capacity of stem cells. The human TERT gene is highly expressed in induced pluripotent stem cells (iPSCs), but not somatic cells. Therefore, activation of the TERT gene is essential for reprogramming human somatic cells into iPSCs [1].

Reprogramming is accompanied with genome-wide epigenetic modifications. Genomic DNA methylation, one mode of epigenetic regulation, serves as an important mechanism in development and cell differentiation [2-5] as well as reprogramming in iPSCs. Stem cell marker genes, such as octamer-binding transcription factor $4(O C T-4)$ [1], homeobox transcription factor NANOG (NANOG) [1] and SAL-like 4 (SALL4) [6], show tissue-specific demethylation corresponding to their expression during reprogramming. For instance, the $\mathrm{CpG}$ sites in the OCT-4 promoter are kept hypomethylated, which permits its expression in pluripotent stem cells. In contrast, hypermethylation of the $O C T$-4 promoter silences its expression in somatic cells. Therefore, the DNA methylation profiles of the OCT-4 promoter have been used as an epigenetic marker of iPSC reprogramming $[1,7]$. 
Although TERT is a pluripotency marker gene in humans, the epigenetic regulation of human TERT gene by DNA methylation remains unclear.

In this study, we identified a differentially methylated region in the distal promoter of the human TERT gene. Hypermethylation of the TERT promoter induced its expression. We show here a novel epigenetic regulatory mechanism wherein hypermethylation of the TERT promoter was activated through binding to the nuclear membrane.

\section{Materials and methods}

\section{Human cell culture}

Human endometrial (UtE1104) [8], amniotic (AM936EP) [9], menstrual blood cell (Edom22) [8], and fetal lung fibroblast (MRC-5) [10] cells were maintained in Poweredby 10 medium (GlycoTechnica, Yokohama, Japan). Placental artery endothelial cells (PAE551) [9] were cultured in medium from the EGM-2MV BulletKit (Lonza, Walkersville, MD, USA) containing 5\% fetal bovine serum. The human embryonal carcinoma NCR-G3 and NCR-G4 cell lines were cultured in G031101 medium, as previously described [11]. The human iPSC lines, MRC-iPS-25 [12], PAE-iPS-1 [13], AM-iPS-8 [9], UtE-iPS-6 [13], EdomiPS-2 [13], and iPS-201B7 [1] were derived from the MRC-5, PAE551, AM963EP, UtE1104, Edom22 and HDF cell lines, respectively. iPSCs were maintained on irradiated mouse embryonic fibroblasts in KO-DMEM (Thermo Fisher Scientific, Inc., Waltham, MA, USA) containing 20\% knockout-serum replacement (Thermo), supplemented with 1\% GlutaMAX (Thermo), 1\% nonessential amino acids (Thermo), $55 \mu \mathrm{M}$ 2-mercaptoethanol (Thermo), 1\% penicillin and streptomycin (Thermo) and $10 \mathrm{ng} / \mathrm{ml}$ recombinant human basic fibroblast growth factor (Wako Pure Chemical Industries, Ltd., Osaka, Japan). Frozen pellets of human embryonic stem cell lines (HUESCs) [14] were kindly gifted from Drs. C. Cowan and T. Tenzan (Harvard Stem Cell Institute, Harvard University, Cambridge, MA). For demethylation assays, MRC-iPSCs were cultured for 4 days in medium containing $0,0.5,1$, or $5 \mu \mathrm{M} 5$-aza-2'-deoxycytidine (5-aza$\mathrm{dC}$, Merck Millipore, Billerica, MA, USA). The human cells used in this study are summarized in Supplemental Table 1.

\section{Gene expression analysis}

For the reverse transcription-polymerase chain reaction (RTPCR), first-strand cDNA was synthesized using total RNA $(1 \mu \mathrm{g})$ with random hexamers and ReverTra Ace reverse transcriptase (TOYOBO Co., Ltd., Osaka, Japan). The cDNA template was amplified using BIOTAQ ${ }^{\mathrm{TM}}$ HS DNA Polymerase (Bioline Ltd; London, UK) and specific primers for TERT, POU5F1, NANOG, SOX2, SALL4, EPHA1, and $G A P D H$. All PCR experiments were performed under the following thermocycling conditions: $95{ }^{\circ} \mathrm{C}$ for $10 \mathrm{~min} ; 30$ cycles of $95{ }^{\circ} \mathrm{C}$ for $30 \mathrm{~s}, 60{ }^{\circ} \mathrm{C}$ for $30 \mathrm{~s}$, and $72{ }^{\circ} \mathrm{C}$ for $1 \mathrm{~min}$; with a final extension at $72{ }^{\circ} \mathrm{C}$ for $10 \mathrm{~min}$. Quantitative realtime PCR (qPCR) was performed using SYBR ${ }^{\circledR}$ Green PCR master mix (Applied Biosystems, Waltham, MA, USA). Data were normalized to $G A P D H$ expression. Gene expression levels are presented as the fold-change in expression, which was calculated using the $2^{-\Delta \Delta C T}$ method [15]. The sequences of primers used in this study are summarized in Supplemental Table 2.

Gene expression array data were obtained from Gene Expression Omnibus microarray data archive (GEO; http://www.ncbi.nlm.nih.gov/geo/) (accession numbers GSE20750, GSE42099, and GSE24677) [13, 16]. All expression data had been analyzed using the same platform, namely an Agilent-014850 Whole Human Genome Microarray $4 x 44 \mathrm{~K} \mathrm{G} 4112 \mathrm{~F}$. The expression data were processed by median normalization, then used for our analysis.

\section{Genome-wide DNA methylation analysis}

Genome-wide DNA methylation analysis was performed using the Illumina Infinium HumanMethylation450K BeadChip Kit (Illumina Inc., San Diego, CA, USA) and the BeadChip was scanned on a BeadArray Reader (Illumina Inc.) according to the manufacturer's instructions. Methylated and unmethylated signals were used to compute a $\beta$ value, which was a quantitative score of the DNA methylation levels, ranging from "0.00" (completely unmethylated) to "1.00" (completely methylated). All DNA methylation data were submitted to GEO under accession numbers GSE73938 and GSE99716.

\section{Bisulfite sequencing}

Sodium bisulfite treatment of genomic DNA was performed using the EZ DNA Methylation-Gold ${ }^{\mathrm{TM}}$ Kit (Zymo Research, Irvine, CA). PCR amplification was performed using BIOTAQ ${ }^{\mathrm{TM}}$ HS DNA Polymerase (Bioline Ltd) and specific primers for TERT promoter. All PCR experiments were performed under the following thermocycling conditions: $95^{\circ} \mathrm{C}$ for $10 \mathrm{~min}$; 40 cycles of $95^{\circ} \mathrm{C}$ for $30 \mathrm{~s}, 55^{\circ} \mathrm{C}$ for $30 \mathrm{~s}$, and $72{ }^{\circ} \mathrm{C}$ for $1 \mathrm{~min}$; with a final extension at $72{ }^{\circ} \mathrm{C}$ for $10 \mathrm{~min}$. The sequences of primers used in this study are summarized in Supplemental Table 2. To determine the methylation states of individual $\mathrm{CpG}$ site at the TERT promoter, the PCR product was gel-extracted, subcloned into the pGEM-T Easy vector (Promega, Madison, WI), and sequenced. Methylation sites were visualized, and quality control was performed using the web-based tool, "QUMA" (http://quma.cdb.riken.jp/) [17]. 


\section{Region-specific methylated $T E R T$ reporter assay}

The 5'-flanking fragment of the human TERT gene was isolated from genomic DNA of MRC-iPSCs by genomic PCR. The position of the transcription start site (TSS) was designated as +1 . The 1.6-kbp, 5'-flanking region (from - 1560 to + 36) containing an EcoRI site at - 625 nucleotides (nt) was cloned into pGL4.10-Basic vector (Promega). This construct was designated as TERT-1560. The TERT626 construct was generated by removing the region from - 1560 to -625 from TERT-1560, following digestion with Acc65I [New England BioLabs (NEB), Beverly, MA] and EcoRI (NEB), and self-ligation. The TERT-1560_Me construct was generated by re-ligation of the fragment (from - 1560 to -625 ) that was methylated in vitro with SssI methylase (NEB). MRC-5 cells and MRC-iPSCs were cotransfected with the reporter constructs and pGL4.74[hRluc/ TK], at a 6: 1 molar ratio in each case. Luciferase activities were determined using the Dual-Luciferase Reporter Assay system (Promega) at $48 \mathrm{~h}$ post-transfection, according to the manufacturer's instructions. Promoter activity was normalized to Renilla luciferase activity. Assays were performed at least 3 times independently.

\section{Chromatin immunoprecipitation (ChIP) assay}

The cells were fixed with $1 \%$ formaldehyde for $10 \mathrm{~min}$ at room temperature and then incubated in $125 \mathrm{mM}$ glycine for $10 \mathrm{~min}$, to quench reactive aldehydes. The cells were collected after rinsing them with phosphate-buffered saline (PBS). The cells were lysed in lysis buffer [10 mM Tris-HCl ( $\mathrm{pH} 8.0$ ), $10 \mathrm{mM} \mathrm{NaCl}$, and $0.2 \%$ Triton-X] and sonicated until the chromatin fragments became 200-1000 bp in size. The antibodies used for ChIP were as follows: anti-trimethylated histone H3 Lys 4 (H3K4me3) antibody (\#MABI0004: MAB Institute Inc., Sapporo, Japan), anti-trimethylated histone H3 Lys 27 (H3K27me3) antibody (\#17-622: Merck Millipore), anti-HP-1 $\alpha$ antibody (\#2616: CST Inc., Danvers, MA, USA), and anti-Lamin B1antibody (\#ab16048: Abcam, Cambridge, UK). Rabbit IgG (\#PP64B: Merck Millipore) and mouse IgG (\#12-371B: Merck Millipore) were used as negative controls to test the specificity of the ChIP results. After the ChIP step, the recovered chromatin samples were subjected to qPCR (ChIP-qPCR) using specific primers (Supplemental Table 2). The percent-input values were calculated as described previously [18]. The qPCR data for each primer pair were represented as percent-input by calculating each specific DNA fragment in immunoprecipitates relative to that in input DNA. The final values were determined by subtraction of the percent-input value obtained for a negative control immunoprecipitation with rabbit $\mathrm{IgG}$ or mouse IgG from the percent-input value of interest.

\section{Statistical analysis}

Differences between 2 independent samples were evaluated by performing two-tailed Student's $t$ test. All error bars represent the standard error of mean (SEM). Linear regression analysis was used to analyze correlations between gene expression and DNA methylation.

\section{Results and discussion}

\section{Identification of a differentially methylated region of the human TERT gene}

Initially, we confirmed TERT expression in human embryonic stem cells (ESCs), embryonal carcinoma cells (ECCs), and iPSCs and their parental somatic cells. The TERT gene was expressed in pluripotent stem cells including ESCs, ECCs, and iPSCs, but was not detected in any of the parental somatic cells (Fig. 1a).

Analysis of DNA methylation rates around the TERT promoter using DNA methylation array data appeared to indicate that the distal region (probe positions a-c in Fig. 1b) was more higher methylated in iPSCs than in somatic cells, although little difference was found at the proximal region (probe positions d-f in Fig. 1b) between iPSCs and somatic cells (Fig. 1b). Bisulfite sequencing analysis was performed to further investigate the DNA methylation state of individual $\mathrm{CpG}$ sites around the TERT promoter from positions -2056 to $+46 \mathrm{nt}$, which contains $123 \mathrm{CpG}$ sites. Regardless of TERT expression and the cell types, the $\mathrm{CpG}$ sites located between -610 and $+46 \mathrm{nt}$ were rarely methylated and those located between - 2056 and - 1566 nt were highly methylated. However, the $\mathrm{CpG}$ sites from - 1415 to -638 nt showed cell type-specific differential methylation. The methylation rates at this region in somatic cells were 20.29, 29.71, 34.12, 40.59, and 36.18\% in AM936EP, Edom22, MRC-5, PAE551, and UtE1104 cells, respectively. In contrast, the methylation rates in pluripotent stem cells were 88.24, 92.94, 89.71, 80.88, and 72.06\% in MRC-iPS-25, PAE-iPS-1, UtE-iPS-6, HUES-8 and NCR-G3 cells, respectively (Fig. 2a). This region includes $34 \mathrm{CpG}$ sites that was highly methylated in ESCs, iPSCs, and ECCs expressing the TERT gene, but was hypomethylated in somatic cells lacking TERT expression. We designated this region as TERT-DMR (TERT differentially methylated region).

To gain insight into the correlation between gene expression and DNA methylation, a scatter plot of gene expression versus DNA methylation with pluripotent marker genes including POU5F1 (also known as OCT-4), SALL4, DPPA4, and TERT of 2 ESCs, 6 iPSCs, and the parental somatic cells were plotted using array data for gene expression and DNA methylation. As expected, POU5F1, SALL4, and 


\section{a}

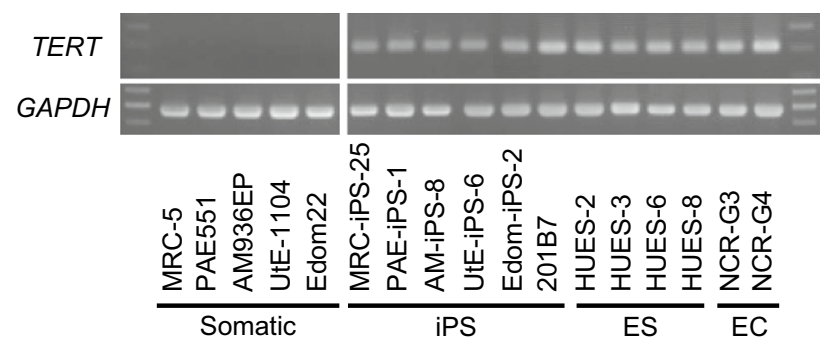

b

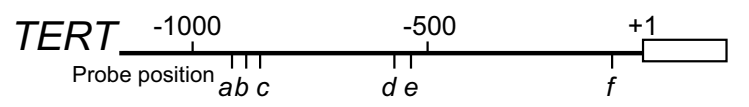

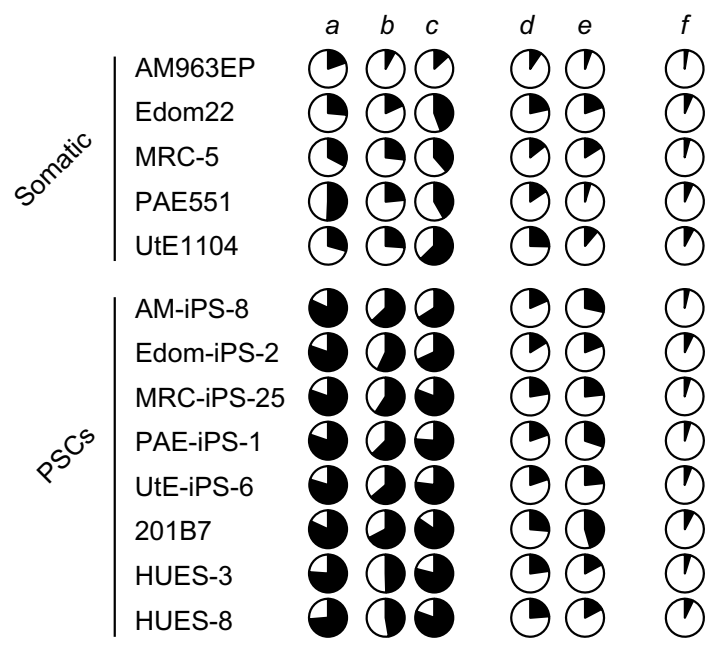

Fig. 1 Expression of the human TERT gene and DNA methylation of the TERT promoter region. a Expression of the TERT gene in 5 somatic, 6 iPSC, 4 ESC, and 2 ECC lines, as detected by RT-PCR. The upper and lower panels show TERT and GAPDH expression, respectively. b DNA methylation rate at the TERT promoter region in parental somatic cells and pluripotent stem cells. (Top) diagram of the TERT gene. The white box indicates the 1st exon of the TERT gene. The position of the transcription start site (TSS) was designated as +1 . Black vertical lines $(a-f)$ indicate the position of each probe. The probe identification numbers of a- $\mathrm{f}$ are $\operatorname{cg} 07380026(-845)$, cg26006951 (- 826), cg17166338 (- 807), cg10767223 (- 637), cg11625005 (- 575), and cg10896616 (- 105), respectively. (Bottom) DNA methylation levels were determined with the Illumina Infinium HumanMethylation450K assay. The relative ratios of methylated and unmethylated DNA are indicated by the black and white areas, respectively, in the pie chart. PSCs, pluripotent stem cells

DPPA4 showed negative correlations (Fig. $2 \mathrm{~b} ; R^{2}=0.8428$, $0.9745,0.8039$, respectively) between DNA methylation and gene expression by linear regression analysis. These results indicated that POU5F1, SALL4, and DPPA4 with hypomethylated promoters were highly expressed in ESCs and iPSCs, whereas low expression of those genes with hypermethylated promoters occurred in the parental somatic cells. Interestingly, TERT showed positive correlation (Fig. $2 \mathrm{~b} ; R^{2}=0.9117$ ) between gene expression and
DNA methylation at the distal promoter region, suggesting that in ESCs and iPSCs, TERT was highly expressed from hypermethylated promoters and that in the parent somatic cells, TERT were poorly expressed from hypomethylated promoters. These results suggested the TERT expression is restricted in pluripotent stem cells and that TERT expression might be regulated by DNA methylation in manner different than other pluripotent marker genes.

\section{Demethylation led to suppression of the human TERT gene}

To investigate whether TERT expression was regulated by the DNA methylation status of the TERT-DMR, we conducted demethylation assay using MRC-iPSCs. In the demethylation assay, MRC-iPSCs were treated with a demethylating reagent (5-aza-dC) for 4 days, and TERT expression was measured. Treatment with 5 -aza-dC caused significant demethylation at the TERT-DMR (Supplemental Fig. 1) and significant suppression of the TERT gene in iPSCs (Fig. 3). Expression of typical stem cell marker genes, POU5F1, NANOG, SOX2, SALL4, and EPHA1 was still maintained in 5-aza-dC-treated iPSCs (Fig. 3). These results suggested that hypermethylation of the TERT-DMR is important for maintaining TERT expression in iPSCs.

\section{Methylation at the TERT-DMR enhanced promoter activity}

To further analyze of the effect of DNA methylation at the TERT-DMR on TERT transcription, region-specific methylated-promoter assays were performed using MRCiPSCs and the parental somatic cells. We designed four reporter constructs: empty vector (pGL4.10), TERT-626, TERT-1560, and TERT-1560_Me. The DNA sequences of TERT-1560 and TERT-1560_Me were identical, with the only difference being in their DNA methylation states. The TERT-626 construct, containing the TERT-proximal region but not the TERT-DMR, showed a significant increase in luciferase activity relative to that of the empty pGL4.10 vector in iPSCs. The TERT-1560 construct showed significantly less reporter activity relative to TERT-626, being comparable to pGL4.10. Interestingly, the TERT-1560_Me construct showed significantly higher reporter activity than the unmethylated construct, TERT-1560 (Fig. 4). In the parental somatic cells (MRC-5), none of the constructs showed significantly increased activity relative to that of the pGL4.10. These results suggested that DNA hypermethylation of the TERT-DMR was indispensable for TERT expression and that he TERT-DMR included an expression regulatory element.

Combining the results of the demethylation and methylation assays revealed the possibility that the TERT-DMR contains a region that suppresses TERT expression. Thus, 
Fig. 2 Bisulfite sequencing analysis and correlation between DNA methylation and gene expression. a Bisulfite sequencing analysis of the 5 '-flanking regions of the TERT gene. (Top) diagram of the TERT gene. The white box indicates the 1st exon of the TERT gene. Black vertical lines indicate the position of individual $\mathrm{CpG}$ sites. The position of the TSS was designated as +1 . Black horizontal lines (T1-T6) indicate the regions studied by bisulfite sequencing. (Bottom) the open and closed circles indicate the unmethylated and methylated states of each CpG site, respectively. Black arrowhead represents the position of the $\mathrm{CpG}$ site in Infinium HumanMethylation450K assay (cg07380026). The horizontal line at the bottom of the figure indicates the defined TERT-

DMR extending from positions - 1415 to -638 nt. b Scatter plot of DNA methylation and gene expression levels for POU5F1, SALL4, DPPA4, and TERT. DNA methylation levels were determined by performing the Illumina Infinium HumanMethylation450K assay. The methylation probes used for POU5F1, SALL4, DPPA4, and TERT (shown in the plot) are cg15948871, cg10941835, cg14836960, and $\operatorname{cg} 07380026$, respectively. White triangles, iPSCs $(n=6)$. White inverted triangles, ESCs $(n=2)$. Black circles, somatic cells $(n=5)$. Black lines, linear regression lines a

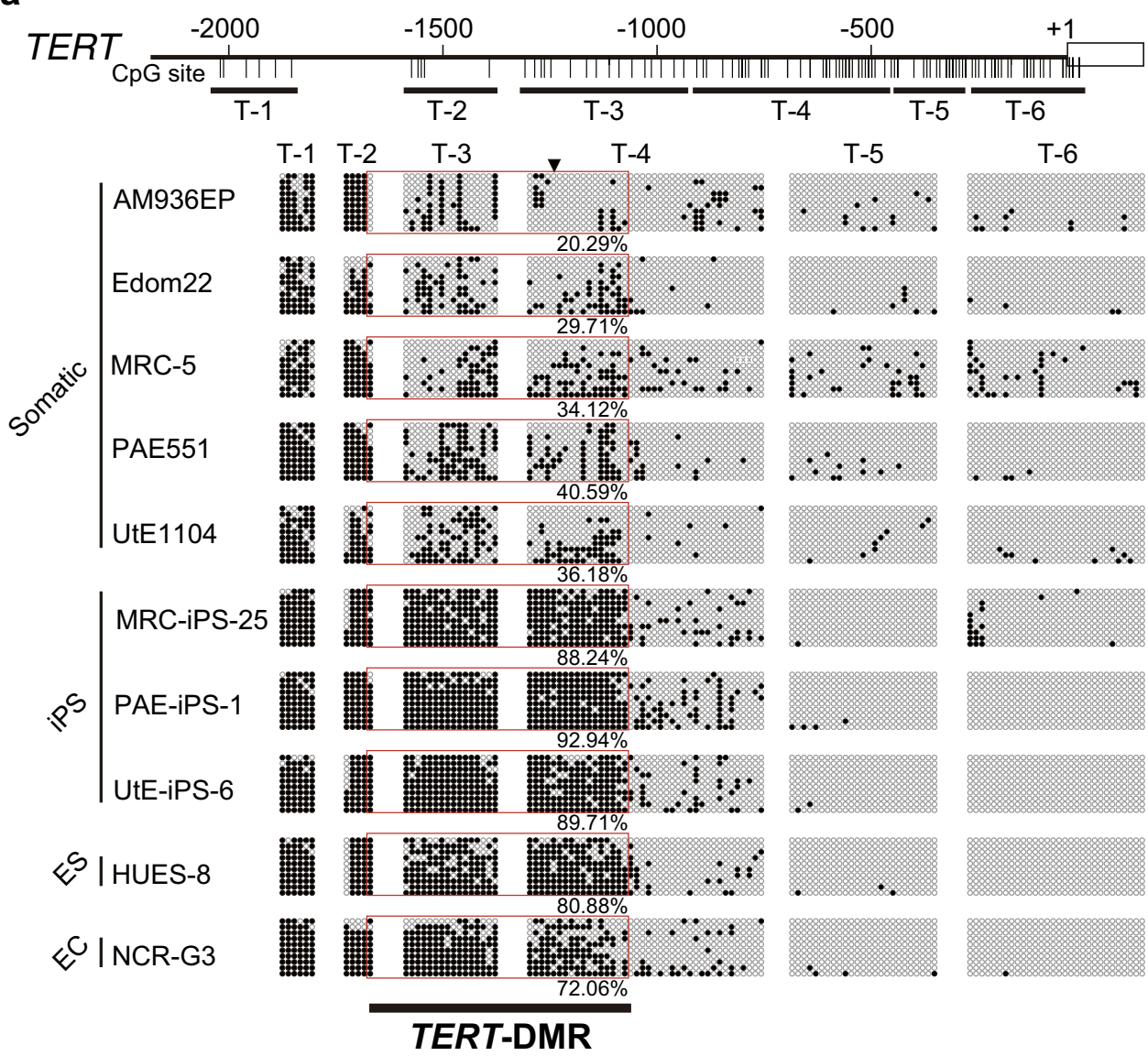

b
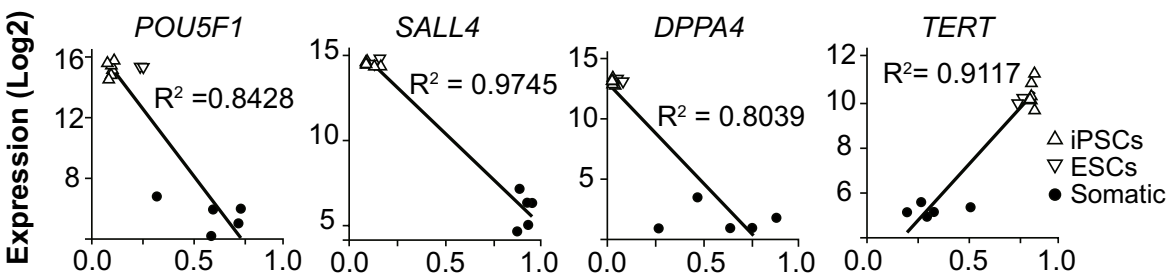

DNA Methylation level we performed electrophoretic mobility shift assay (EMSA) to determine whether hypermethylation of the TERT-DMR prevents certain transcriptional suppressors from binding the TERT-DMR. However, no factors that depend on the methylation status were detected (Supplemental Fig. 2a, b).

\section{Methylated TERT-DMR maintained open chromatin}

Histone modification is another epigenetic mechanism for regulating gene expression. Trimethylated histone H3 Lys 4 (H3K4me3) tends to distribute in "active" gene loci [19], and distribute in hypomethylated DNA region [20]. In contrast, trimethylated histone H3 Lys 27 (H3K27me3) tends to distribute in "inactive" gene loci [19]. In addition, inactive gene loci are generally enriched for the heterochromatic protein HP- $1 \alpha$ and form heterochromatin. The genomic DNA in heterochromatin is usually hypermethylated [21]. Therefore, we performed ChIP-qPCR for H3K4me3, H3K27me3, and HP- $1 \alpha$ to determine the histone modification status at the TERT-DMR. ChIP-qPCR revealed that $\mathrm{H} 3 \mathrm{~K} 4 \mathrm{me} 3$ was significantly enriched in iPSCs, whereas the H3K27me3 and HP- $1 \alpha$ were significantly enriched in somatic cells (Fig. 5a-d). Furthermore, analyzing the dataset of ATACseq analysis [22] of human ESCs (GSM2433022) [23] and human somatic cells (GSM1939273) revealed that the chromatin structure around the TERT-DMR was "open" in $T E R T$-expressing pluripotent cells, but was closed in somatic cells. These data indicated that histones at the TERT-DMR 


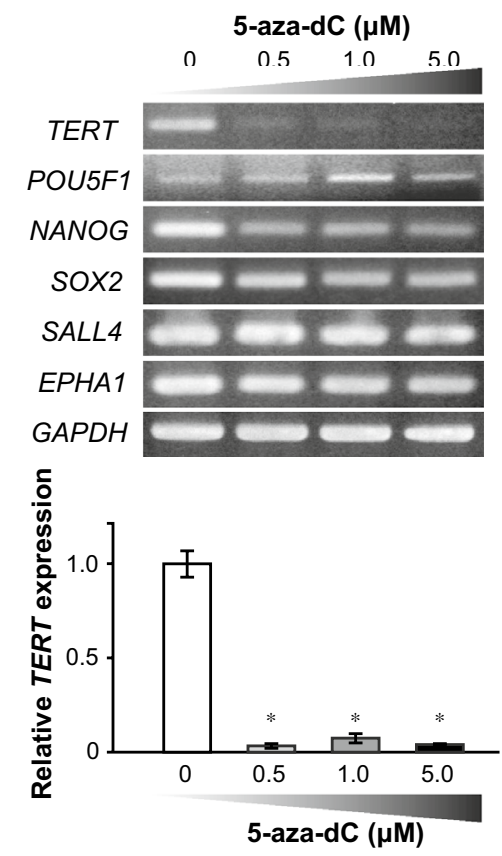

Fig. 3 Demethylation of the TERT-DMR led to suppression of TERT expression. Effect of a demethylation reagent (5-aza-dC) on the expression of TERT and pluripotent marker genes in MRC-iPSCs. (Top) TERT, POU5F1, NANOG, SOX2, SALL4, EPHA1, and GAPDH gene expression. GAPDH was detected as an internal control. (Bottom) relative TERT gene expression levels measured by $\mathrm{qPCR}$, with normalization to GAPDH expression using the delta-delta CT method. The data shown represent the mean \pm SE. $* p<0.01$

were active state, even though the DNA was highly methylated in human iPSCs.

\section{Role of hypermethylation of the TERT-DMR in regulating TERT expression}

In human iPSCs that express the TERT gene, the TERTDMR was hypermethylated under the active state of histone modification. These results suggested that hypermethylation of the TERT-DMR promoted TERT expression. To clarify the role of hypermethylation at the TERT-DMR in regulating $T E R T$ gene expression, we searched for factors that interact with the TERT-DMR. First, we performed chromosome conformation capture (3C) and Splinkerrete-3C (S3C) assays (see Supplementary methods) to determine whether hypermethylation of the TERT-DMR affects the genomic interaction between the TERT-DMR and other genomic loci. No genomic interaction was detected (data not shown).

Next, we focused on the nuclear lamina. The nuclear lamina is a polymeric protein meshwork associated with the nucleoplasmic surface of the inner nuclear membrane [24, 25 ] that has been associated with gene silencing by interacting with genomic DNA $[26,27]$. We performed ChIP-qPCR for Lamin B1, which is a representative component of the

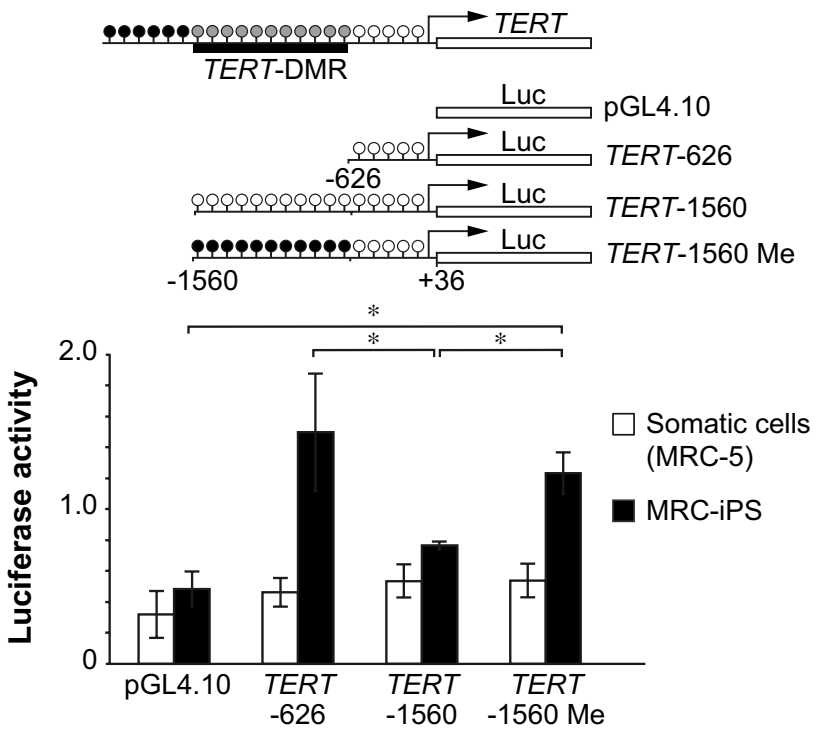

Fig. 4 Methylation of the TERT-DMR enhanced the promoter activity. Region-specific methylated-reporter assay in MRC-iPSCs and parental somatic cells (MRC-5). (Top) schematic representation of the TERT gene and reporter constructs. Open and closed circles indicate unmethylated and methylated $\mathrm{CpG}$ sites in the constructs, respectively. (Bottom) luciferase activity of each reporter construct. White bars indicate luciferase activities in parent somatic cells (MRC-5). Black bars indicate luciferase activities in MRC-iPSCs. Each bar shown in the figure represents the mean \pm SEM. $* p<0.05$

nuclear lamina, to determine whether hypermethylation of the TERT-DMR inhibited binding to the nuclear lamina. Unexpectedly, Lamin B1 was enriched at the TERT-DMR in iPSCs, but not in somatic cells (Fig. 5e), meaning that Lamin B1 was selectively interacted with methylated TERT-DMR.

Our findings suggested the model whereby human TERT expression is regulated: in the TERT-expressing cells such as iPSCs and ESCs, the TERT-DMR is highly methylated and binds to the nuclear lamina via Lamin B1, which lead to an open chromatin structure, enabling transcription factors, such as c-MYC [28, 29], to bind the proximal promoter and drive TERT expression. In contrast, in cells not expressing TERT such as most somatic cells, the unmethylated TERTDMR is free in the nucleus. H3K27me 3 and HP- $1 \alpha$ become enriched at the unmethylated TERT-DMR, forming heterochromatin (Fig. 6).

Several groups have discussed the epigenetic regulation of the human TERT gene [30-33]; however, no clear relationship between DNA methylation and TERT expression has been demonstrated. One reason why the epigenetic regulation of TERT has been poorly understood may be that previous studies were focused on the proximal core promoter region (approximately 500-bp upstream from the TSS). In this study, we focused on the distal promoter region of the $T E R T$ gene and identified the TERT-DMR, which provides additional information for the human TERT gene. 
a

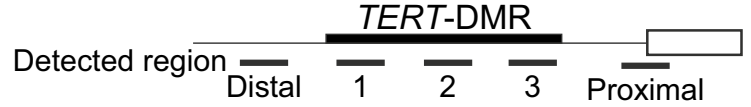

b

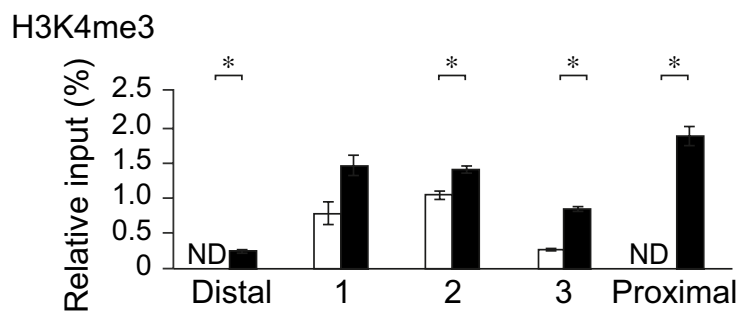

C

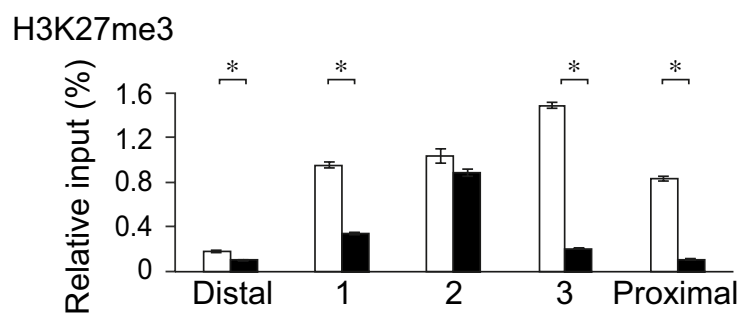

d $\mathrm{HP}-1 \alpha$

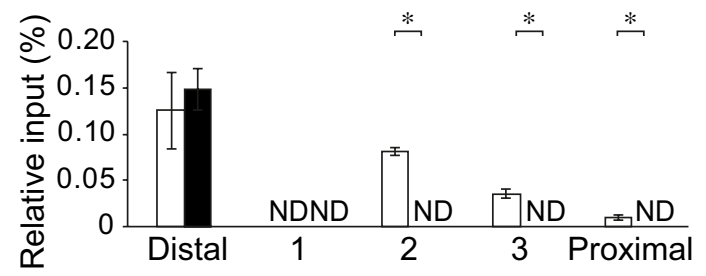

e Lamin B1

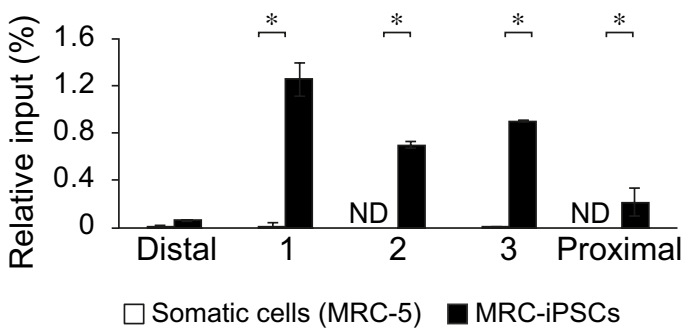

Fig. 5 ChIP-qPCR at the $T E R T$-DMR and neighboring region. a Schematic diagram of the 5 '-flanking region of TERT. Horizontal black bars indicate regions targeted by ChIP-qPCR. The white box indicates the 1st exon of the TERT gene. ChIP-qPCR for H3K4me3 (b), H3K27me3 (c), HP-1 $\alpha$ (d), and Lamin B1 (e) in somatic cells (MRC-5) and MRC-iPSCs. Enrichment at each position is indicated as normalized percent-input (IgG-subtracted). The white and black bars indicate data from parent somatic cells and iPSCs, respectively. Each bar shown in the figure represents the mean \pm SEM. ${ }^{*} p<0.05$

Of note, the demethylating reagent 5 -aza-dC (also known as decitabine) has been used clinically for treating acute myeloid leukemia (AML) in patients whose malignant cells express TERT gene [34]. In addition, treatment with decitabine has been reported to suppress expression and inhibit malignant cell proliferation [35, 36]. However,

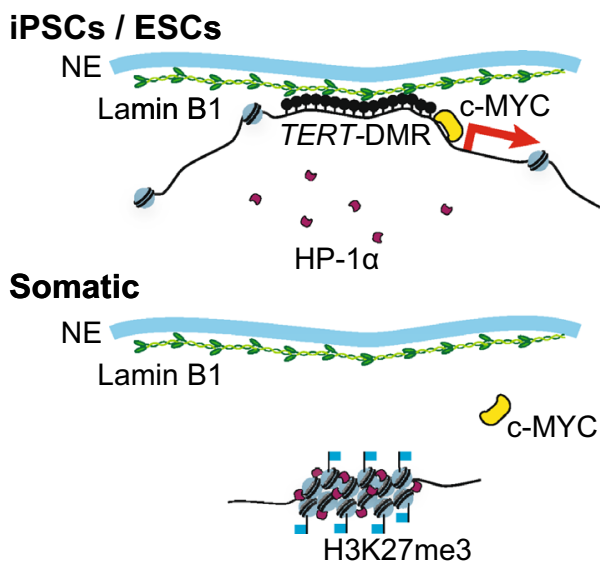

Fig. 6 Model of TERT expression regulated by TERT-DMR hypermethylation. Schematic representation shown our proposed model whereby TERT expression is regulated by hypermethylation of the TERT-DMR. (Top) in TERT-expressing cells, such as iPSCs and ESCs, the TERT-DMR is highly methylated and binds to Lamin B1, a component of the nuclear lamina, which leads to an open chromatin structure and enables c-MYC to bind the proximal promoter for TERT expression. (Bottom) in cells not expressing TERT, such as most somatic cells, the unmethylated TERT-DMR is free in the nucleus. H3K27me 3 and HP-1 $\alpha$ become enriched at the unmethylated TERTDMR, forming heterochromatin

exactly how decitabine suppresses TERT expression remains unclear. In this study, demethylating TERT-DMR with 5-aza-dC suppressed TERT expression. Therefore, the therapeutic effect of decitabine in AML potentially involves the inhibition of TERT expression through TERTDMR demethylation. Moreover, monitoring DNA methylation at the TERT-DMR may be useful for diagnosis, prognosis, and as a biomarker for estimating the risk for developing AML.

In conclusion, we identified TERT-DMR at the distal $T E R T$ promoter region. Hypermethylation of the TERTDMR promoted activation of TERT gene expression by binding to the nuclear membrane. Our findings shed light on a new functional aspect of DNA methylation with respect to gene expression. This novel epigenetic regulation mechanism is required for the reprogramming the transformation of somatic cells into iPSCs.

Acknowledgements We would like to express our sincere thanks to Drs. C. Cowan and T. Tenzan for the HUESC lines; to Drs. N. Hattori and J. Tomikawa for assistance with the ChIP and 3C method, respectively; to Dr. S. Wakitani for discussion. This study was funded by a Grant-in-Aid for the Japan Society for the Promotion of Science (JSPS) Research Fellow (no. JP15J11596 to K. T.), a Grant-in-Aid for Scientific Research (B) (no. 25292187 to K. N.), and a Grant-in-Aid for challenging Exploratory Research (no. 16K15055 to K. N.) from the JSPS KAKENHI; and by funding to K. N. from The NOVARTIS Foundation (Japan) for the Promotion of Science, the Terumo Foundation grant, and the Takeda Science Foundation. 


\section{Compliance with ethical standards}

Conflict of interest The authors declare that they have no conflict of interest.

Ethical approval This study was approved by the research ethics committee of University of Miyazaki and Institutional Review Board of the National Institute for Child Health and Development. All procedures performed in studied involving the handling of human cells were in accordance with the ethical standards of the 1964 Helsinki declaration and its later amendments or comparable ethical standard.

Open Access This article is distributed under the terms of the Creative Commons Attribution 4.0 International License (http://creativecommons.org/licenses/by/4.0/), which permits unrestricted use, distribution, and reproduction in any medium, provided you give appropriate credit to the original author(s) and the source, provide a link to the Creative Commons license, and indicate if changes were made.

\section{References}

1. Takahashi K, Tanabe K, Ohnuki M, Narita M, Ichisaka T, Tomoda $\mathrm{K}$, et al. Induction of pluripotent stem cells from adult human fibroblasts by defined factors. Cell. 2007;131:861-72.

2. Imamura $T$, Ohgane J, Ito S, Ogawa T, Hattori N, Tanaka S, et al. $\mathrm{CpG}$ island of rat sphingosine kinase-1 gene: tissuedependent DNA methylation status and multiple alternative first exons. Genomics. 2001;76:117-25.

3. Shen CK, Maniatis T. Tissue-specific DNA methylation in a cluster of rabbit beta-like globin genes. Proc Natl Acad Sci. 1980;77(11):6634-8.

4. Ko YG, Nishino K, Hattori N, Arai Y, Tanaka S, Shiota K. Stage-by-stage change in DNA methylation status of Dnmt 1 locus during mouse early development. J Biol Chem. 2005;280:9627-34.

5. Nishino K, Hattori N, Tanaka S, Shiota K. DNA methylationmediated control of Sry gene expression in mouse gonadal development. J Biol Chem. 2004;279:22306-13.

6. Nishino K, Toyoda M, Yamazaki-Inoue M, Makino H, Fukawatase Y, Chikazawa E, et al. Defining hypo-methylated regions of stem cell-specific promoters in human iPS cells derived from extra-embryonic amnions and lung fibroblasts. PLoS One. 2010;5:e13017.

7. Takahashi K, Yamanaka S. Induction of pluripotent stem cells from mouse embryonic and adult fibroblast cultures by defined factors. Cell. 2006;126:663-76.

8. Cui C-H, Uyama T, Miyado K, Terai M, Kyo S, Kiyono T, et al. Menstrual blood-derived cells confer human dystrophin expression in the murine model of Duchenne muscular dystrophy via cell fusion and myogenic transdifferentiation. Mol Biol Cell. 2007;18:1586-94

9. Nagata S, Toyoda M, Yamaguchi S, Hirano K, Makino H, Nishino K, et al. Efficient reprogramming of human and mouse primary extra-embryonic cells to pluripotent stem cells. Genes Cells. 2009; 14:1395-404.

10. Jacobs JP, Jones CM, Baille JP. Characteristics of a human diploid cell designated MRC-5. Nature. 1970;227:168-70.

11. Hata J, Fujimoto J, Ishii E, Umezawa A, Kokai Y, Matsubayashi $\mathrm{Y}$, et al. Differentiation of human germ cell tumor cells in vivo and in vitro. Acta Histochem Cytochem. 1992;25:563-76.
12. Makino H, Toyoda M, Matsumoto K, Saito H, Nishino K, Fukawatase Y, et al. Mesenchymal to embryonic incomplete transition of human cells by chimeric OCT4/3 (POU5F1) with physiological co-activator EWS. Exp Cell Res. 2009;315:2727-40.

13. Nishino K, Toyoda M, Yamazaki-Inoue M, Fukawatase Y, Chikazawa E, Sakaguchi H, et al. DNA methylation dynamics in human induced pluripotent stem cells over time. PLoS Genet. 2011;7:e1002085.

14. Cowan CA, Klimanskaya I, McMahon J, Atienza J, Witmyer J, Zucker JP, et al. Derivation of embryonic stem-cell lines from human blastocysts. N Engl J Med. 2004;350:1353-6.

15. Livak KJ, Schmittgen TD. Analysis of relative gene expression data using real-time quantitative PCR and the 2(-Delta Delta $\mathrm{C}(\mathrm{T})$ ) method. Methods. 2001;25:402-8.

16. Tateno H, Matsushima A, Hiemori K, Onuma Y, Ito Y, Hasehira $\mathrm{K}$, et al. Podocalyxin Is a glycoprotein ligand of the human pluripotent stem cell-specific probe rBC2LCN. Stem Cells Transl Med. 2013;2:265-73.

17. Kumaki Y, Oda M, Okano M. QUMA: quantification tool for methylation analysis. Nucleic Acids Res. 2008;36:W170-5. 10.1093/nar/gkn294.

18. Yellajoshyula D, Lim J, Thompson DM, Witt JS, Patterson ES, Kroll KL. Geminin regulates the transcriptional and epigenetic status of neuronal fate-promoting genes during mammalian neurogenesis. Mol Cell Biol. 2012;32:4549-60.

19. Bracken AP, Dietrich N, Pasini D, Hansen KH, Helin K. Genome-wide mapping of Polycomb target genes unravels their roles in cell fate transitions. Genes Dev. 2006;20:1123-36.

20. Lee D-S, Shin J-Y, Tonge PD, Puri MC, Lee S, Park H, et al. An epigenomic roadmap to induced pluripotency reveals DNA methylation as a reprogramming modulator. Nat Commun. 2014;5:5619.

21. Rose NR, Klose RJ. Understanding the relationship between DNA methylation and histone lysine methylation. BBA Gene Regul Mech. 2014;1839:1362-72.

22. Buenrostro JD, Wu B, Chang HY, Greenleaf WJ. ATAC-seq: a method for assaying chromatin accessibility genome-wide. Curr Protoc Mol Biol. 2015;109:21-9.

23. Boulay G, Awad ME, Riggi N, Archer TC, Iyer S, Boonseng WE, et al. OTX2 activity at distal regulatory elements shapes the chromatin landscape of group 3 medulloblastoma. Cancer Discov. 2017;7:288-301.

24. Butin-Israeli V, Adam SA, Goldman AE, Goldman RD. Nuclear lamin functions and disease. Trends Genet. 2012;28:464-71.

25. Dechat T, Adam SA, Taimen P, Shimi T, Goldman RD. Nuclear lamins. Cold Spring Harb Perspect Biol. 2010;2:a000547.

26. Burke B, Stewart CL. The nuclear lamins: flexibility in function. Nat Rev Mol Cell Biol. 2013;14:13-24.

27. Shevelyov YY, Nurminsky DI. The nuclear lamina as a genesilencing hub. Curr Issues Mol Biol. 2012;14:27-38.

28. Horikawa I, Cable PL, Afshari C, Barrett JC. Cloning and characterization of the promoter region of human telomerase reverse transcriptase gene. Cancer Res. 1999;59:826-30.

29. Wu KJ, Grandori C, Amacker M, Simon-Vermot N, Polack A, Lingner J, et al. Direct activation of TERT transcription by c-MYC. Nat Genet. 1999;21:220-4.

30. Guilleret I, Benhattar J. Unusual distribution of DNA methylation within the hTERT CpG island in tissues and cell lines. Biochem Biophys Res Commun. 2004;325:1037-43.

31. Lopatina NG, Poole JC, Saldanha SN, Hansen NJ, Key JS, Pita MA, et al. Control mechanisms in the regulation of telomerase reverse transcriptase expression in differentiating human teratocarcinoma cells. Biochem Biophys Res Commun. 2003;306:650-9. 
32. Guilleret I, Yan P, Grange F, Braunschweig R, Bosman FT, Benhattar J. Hypermethylation of the human telomerase catalytic subunit (hTERT) gene correlates with telomerase activity. Int J Cancer. 2002;101:335-41.

33. Dessain SK, Yu H, Reddel RR, Beijersbergen RL, Weinberg RA. Methylation of the human telomerase gene $\mathrm{CpG}$ island. Cancer Res. 2000;60:537-41.

34. Nieto M, Demolis P, Béhanzin E, Moreau A, Hudson I, Flores B, et al. The European Medicines Agency Review of Decitabine (Dacogen) for the treatment of adult patients with acute myeloid leukemia: summary of the scientific assessment of the committee for medicinal products for human use. Oncologist. 2016;21:692-700.
35. Hájek M, Votruba I, Holý A, Krecmerová M, Tloust'ová E. Alpha anomer of 5-aza-2'-deoxycytidine down-regulates hTERT mRNA expression in human leukemia HL-60 cells. Biochem Pharmacol. 2008;75:965-72.

36. Pettigrew KA, Armstrong RN, Colyer HAA, Zhang S, Rea IM, Jones RE, et al. Differential TERT promoter methylation and response to 5 aza-2'-deoxycytidine in acute myeloid leukemia cell lines: TERT expression, telomerase activity, telomere length, and cell death. Genes Chromosomes Cancer. 2012;780:768-80. 